\title{
Lesiones multiligamentarias de rodilla asociadas a ruptura de tendón rotuliano
}

\author{
Complex multiligament knee injury associated with patellar tendon rupture \\ Canales-Zamora OA,* Benítez-Romero A, ${ }^{\ddagger}$ Mejía-Rohenes LC, \\ Mora-Ríos FG, "González-Gijón OR," Cueva Del Castillo-Mendoza JF**
}

Hospital Regional General «Ignacio Zaragoza», ISSSTE.

RESUMEN. Introducción: La lesión multiligamentaria de la articulación de rodilla se define como el daño a dos o más ligamentos principales. Estas lesiones son poco comunes, con una incidencia de $<10: 10,000$. Constituyen menos de $0.02 \%$ de todas las lesiones ortopédicas. Es aún menos frecuente encontrar asociación de éstas a una ruptura completa del tendón patelar, la cual se presenta con una prevalencia 2.4\%. Caso clínico: Masculino de 22 años sufrió accidente de alta velocidad, con diagnóstico de lesión multiligamentaria de rodilla izquierda estadio V de Schenck asociada a ruptura completa de tendón patelar, fue tratado en un solo tiempo quirúrgico con reconstrucción ligamentaria cuatro semanas después de evento traumático. Se obtuvo a los ocho meses un puntaje de 88.5 con el formulario IKDC 2000 para la evaluación funcional subjetiva de la rodilla. Discusión: El tratamiento quirúrgico definitivo se realiza a las cuatro semanas de la lesión. Con esto se disminuye el riesgo de inestabilidad. En nuestro caso clínico el paciente se logra integrar a las actividades de su vida diaria después de ocho meses. Para validar esta técnica como efectiva o buena para su uso, se requiere un mayor número de pacientes tratados de la misma forma. Sin embargo, se sugiere
ABSTRACT. Introduction: Multi-ligament injury of the knee joint is defined as damage to two or more major ligaments. They are uncommon, with an incidence of $<10: 10,000$. They constitute less than $0.02 \%$ of all orthopedic lesions. It is even less frequent to find an association of these with a complete rupture of the patellar tendon which presents with a prevalence of $2.4 \%$. Case report: 22 year old male; he presented a highspeed accident with a diagnosis of multi-ligament left knee injury stage V of Schenck associated with complete patellar tendon rupture treated in a single surgical time with ligament reconstruction, four weeks after traumatic event. A score of 88.5 was obtained after eight months with the IKDC 2000 form for the subjective functional evaluation of the knee. Discussion: The definitive surgical treatment is performed four weeks after the injury. This reduces the risk of instability. In our clinical case, the patient is integrated into the activities of his daily life after eight months. To validate this technique as effective or good for its use, a greater number of patients treated in the same way is required. It is, however, suggested to contemplate as a management option.

* Médico residente de cuarto año de Ortopedia y Traumatología de la Facultad Mexicana de Medicina, Universidad La Salle.

‡ Médico adscrito al Módulo de Cirugía Articular.

$\S$ Jefe de Servicio.

" Médico adscrito al Módulo Pediátrico.

" Médico residente de cuarto año de Ortopedia y Traumatología, de la Facultad Mexicana de Medicina, Universidad La Salle.

${ }_{*}^{* *}$ Médico adscrito al Módulo de cirugía de columna vertebral.

Hospital Regional General «Ignacio Zaragoza», ISSSTE, México, Ciudad de México.

Correspondencia:

Dr. Óscar Alejandro Canales Zamora

Avenida Zaragoza Núm. 1711,

Col. Ejército Constitucionalista, C.P. 09220 Alcaldía Iztapalapa, CDMX,

E-mail: oskarcanales@hotmail.com

Citar como: Canales-Zamora OA, Benítez-Romero A, Mejía-Rohenes LC, Mora-Ríos FG, González-Gijón OR, Cueva Del Castillo-Mendoza JF. Lesiones multiligamentarias de rodilla asociadas a ruptura de tendón rotuliano. Acta Ortop Mex. 2020; 34(6): 417-421. https://dx.doi.org/10.35366/99141

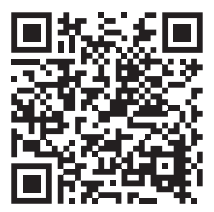


contemplar como una opción de manejo. Conclusión: Lesión poco frecuente, de tratamiento quirúrgico con un pronóstico reservado.

Palabras clave: Lesión, multiligamentaria, patelar, aloinjerto, cirugía.

\section{Introducción}

La lesión multiligamentaria de la articulación de la rodilla se define como el daño a dos o más ligamentos principales: ${ }^{1}$ ligamento cruzado anterior (ACL), ligamento cruzado posterior (PCL), ligamento colateral medial (MCL), ligamento colateral lateral (LCL), esquina posteromedial (PMC) y esquina posterolateral (PLC). ${ }^{2}$ Estas lesiones son poco comunes, se presentan principalmente en pacientes de entre la tercera y cuarta décadas de la vida con mayor frecuencia en el sexo masculino ${ }^{2}$ y están íntimamente relacionadas con luxación de rodilla, con una incidencia de $<$ 10:10,000. ${ }^{2}$ Constituyen menos de $0.02 \%$ de todas la lesiones ortopédicas ${ }^{3}$ y es aún menos frecuente encontrar asociación de éstas a una ruptura del tendón patelar, la cual se presenta con una prevalencia $2.4 \% .{ }^{4}$ Las lesiones multiligamentarias de rodilla asociadas a luxación pueden estar ligadas con lesión de la arteria poplítea (23-32\%), lesión del nervio peroneo (14-40\%), lesión de meniscos (37.3\%), lesiones del cartílago articular en $28.3 \%{ }^{5}$ y las luxaciones expuestas varían desde $9 \%{ }^{6}$ hasta 20 a 35\%. ${ }^{7}$ Más de $60 \%$ se presentan con fracturas. ${ }^{7}$ En la actualidad son difíciles de tratar y hay pocos estudios reportados sobre las mismas.

Son causadas por traumatismos de alta energía, ${ }^{8}$ con excepción de las personas obesas, en las cuales pueden ser causadas por mecanismos de baja velocidad. ${ }^{5}$ La dirección de las fuerzas dicta que los ligamentos se ven afectados: ${ }^{2}$

1. La traslación anterior desgarraría ACL seguido de MCL y LCL. ${ }^{2}$

2. Una traslación posterior sólo afectaría PCL. ${ }^{2}$

3. La aplicación de fuerza en varo desgarraría el LCL seguido de ACL y PCL. ${ }^{2}$

4. La aplicación de fuerza en valgo desgarraría MCL seguido de ACL y PCL.

5. Cuando el fémur se encuentra inmóvil y se aplica una fuerza de rotación interna, se produce desgarro del LCL seguido de PCL y finalmente desgarro de la cápsula articular. ${ }^{2}$

6. Cuando el fémur se encuentra inmóvil y se aplica una fuerza de rotación externa, se produce desgarro MCL seguido de PCL y finalmente desgarro de la cápsula articular. ${ }^{2}$

7. Cuando se aplica una fuerza de hiperextensión, primero habría ruptura PCL seguida de ACL y finalmente de la cápsula posterior.

Evaluación clínica: además de dolor, edema, deformidad anatómica, limitación de los arcos de movilidad, im-
Conclusion: These types of lesions are infrequent with a prognosis reserved.

Keywords: Injury, multi-ligament, patellar, allograft, surgery.

posibilidad para la marcha y el apoyo, el paciente puede referir pérdida de la sensibilidad de la pierna o el pie. La evaluación de los tejidos blandos y la estabilidad de la rodilla así como la evaluación vascular son imperativas. En una luxación de rodilla después de realizar la reducción debe sospechase siempre la lesión multiligamentaria. El examen físico bajo anestesia continúa siendo el más preciso para determinar las lesiones ligamentarias de la rodilla. ${ }^{9}$ Para el diagnóstico imagenológico, las proyecciones radiográficas en estrés son útiles para evaluar la lesiones crónicas, pero éstas son difíciles de realizar en pacientes con lesiones agudas. ${ }^{9}$ La resonancia magnética es el estándar de oro para el diagnóstico de la lesión multiligamentaria de rodilla. ${ }^{2,9} \mathrm{La}$ tendencia actual, apoyada en la literatura, del tratamiento es el quirúrgico para reconstrucción ligamentaria con uso de autoinjerto o aloinjerto. ${ }^{5}$ No obstante, el momento de la cirugía es un tema de debate; algunos autores recomiendan realizar la cirugía dentro de las tres primeras semanas, ya

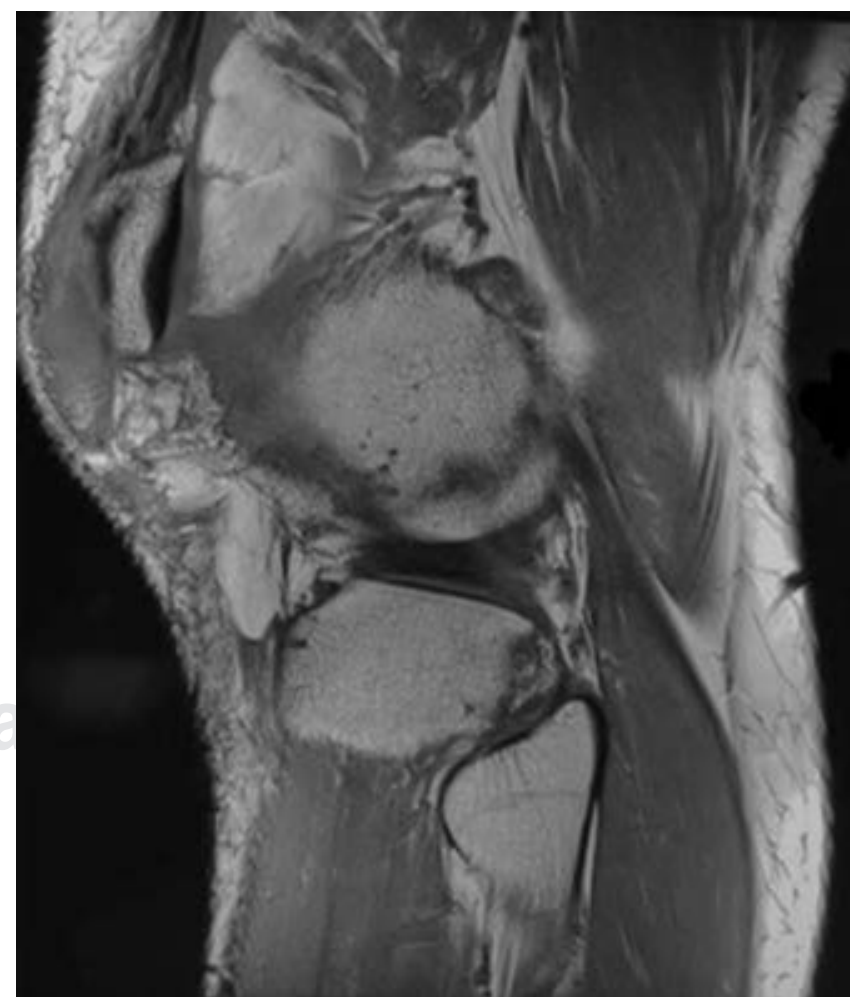

Figura 1: Resonancia magnética, corte sagital T2 con interrupción en la intensidad de la señal a nivel del tendón rotuliano. También se observa interrupción de la señal a nivel de ligamento colateral lateral. 

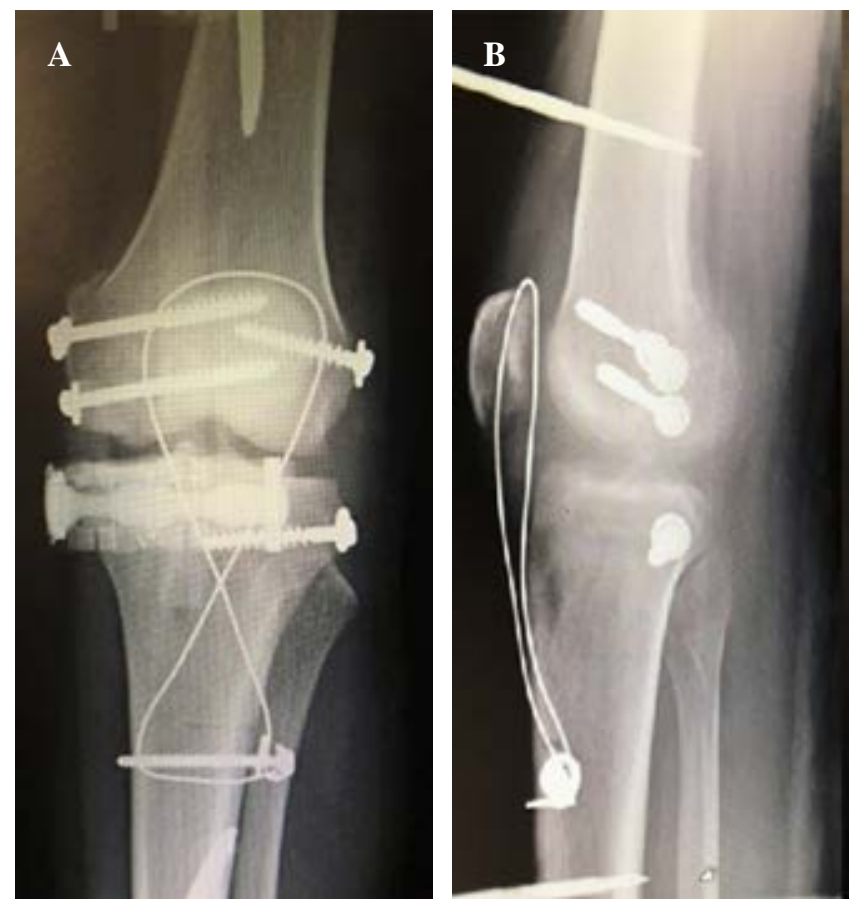

Figura 2: Vista anteroposterior A) y lateral B) de rodilla izquierda. Fijación de aloinjerto para ligamento colateral lateral con tornillos, reducción de cóndilo lateral con dos tornillos de esponjosa, tensor de Muller para protección de la reparación del tendón patelar y fijador externo para protección de reconstrucción ligamentaria.

que es el tiempo crítico para identificar y tratar mejor las estructuras ligamentarias antes de que éstas puedan presentar cicatrización o necrosis tisular. ${ }^{5}$ Esto con el objetivo de reconstruir todas las estructuras lesionadas..$^{9,10}$

\section{Caso clínico}

Masculino de 22 años que sufrió accidente de alta velocidad en motocicleta al viajar como pasajero. Al examen físico de la extremidad pélvica izquierda encontramos luxación expuesta de rodilla izquierda, sangrado abundante, daño a tejidos blandos, a la exploración vascular con Doppler detectamos un índice tobillo-brazo de 1.0 y la exploración neurológica no fue valorable. El paciente inicialmente es tratado en urgencias con protocolo de control de daños, se coloca fijador externo, lavado quirúrgico más desbridamiento mecánico y químico. Durante el procedimiento se detecta ruptura del tendón rotuliano, ligamento cruzado anterior, ligamento cruzado posterior, ligamento poplíteo, ligamento colateral lateral más fractura de cóndilo medial y lesiones condrales en 60\% de la superficie articular del cóndilo medial. La exploración del paquete vascular de la región poplítea sin lesión, con pulsos disminuidos. Se realizó ecografía Doppler de extremidad pélvica izquierda, la cual reporta: 1 . Arteria poplítea permeable, con flujo de aspecto trifásico, 2. Arteria tibial posterior permeable, con flujo de aspecto trifásico, 3. Arteria tibial anterior permeable, con flujo de aspecto trifásico y 4 . Arteria pedia permeable con flujo presente. La resonancia magnética de miembro pélvico izquierdo revela: lesión multiligamentaria en la rodilla más ruptura de tendón rotuliano (Figura 1).

Dadas estas condiciones, decidimos la cirugía de reconstrucción ligamentaria cuatro semanas después del evento traumático. Se realizó con anestesia regional, la herida de la luxación se usó como abordaje con extensión proximal y distal, la ruptura del tendón patelar nos dio una buena exposición de la rodilla. Se utilizaron aloinjertos de tibial posterior y tibial anterior doble para reconstrucción de los ligamentos cruzados. Se inició con túnel tibial del LCP, el alambre guía se colocó aproximadamente $2 \mathrm{~cm}$ distal al túnel tibial del LCA y a la mitad de camino entre la tuberosidad anterior de la tibia y el borde posterior de la tibia (anterolateral). Se elaboraron los túneles femorales para ambos ligamentos cruzados y a través de éstos se pasaron los aloinjertos respectivamente. Se fijó ligamento cruzado posterior en su porción femoral con tornillo biodegradable de $10 \mathrm{~mm}$, a continuación se fijó ligamento cruzado anterior en su porción femoral con tornillo biodegradable de $10 \mathrm{~mm}$, se tensó de forma manual el injerto de ligamento cruzado, posteriormente se fijó en el túnel tibial con tonillo biodegradable de $10 \mathrm{~mm}$ y por último se tensó de forma manual el ligamento cruzado anterior y se fijó en el túnel tibial con tornillo biodegradable de $9 \mathrm{~mm}$.

Para reconstruir el ligamento colateral lateral se usó aloinjerto de peroneo fijado con dos tornillos de esponjosa $6.0 \mathrm{~mm}$ con arandela (Figura 2). El ligamento colateral medial se encontraba íntegro, sólo realizamos reducción de fractura de cóndilo medial con dos tornillos de esponjosa $6.0 \mathrm{~mm}$ con arandela (Figura 2).
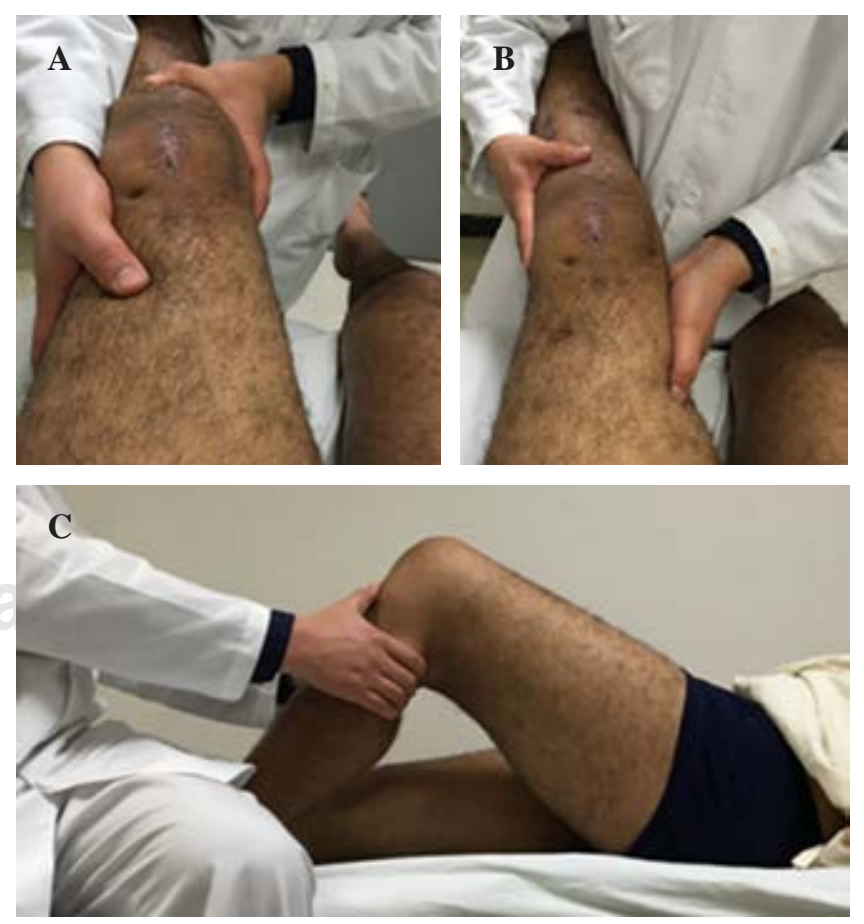

Figura 3: A) Test de estrés en varo negativo. B) Test de estrés en valgo negativo. C) Test cajón anterior negativo. 

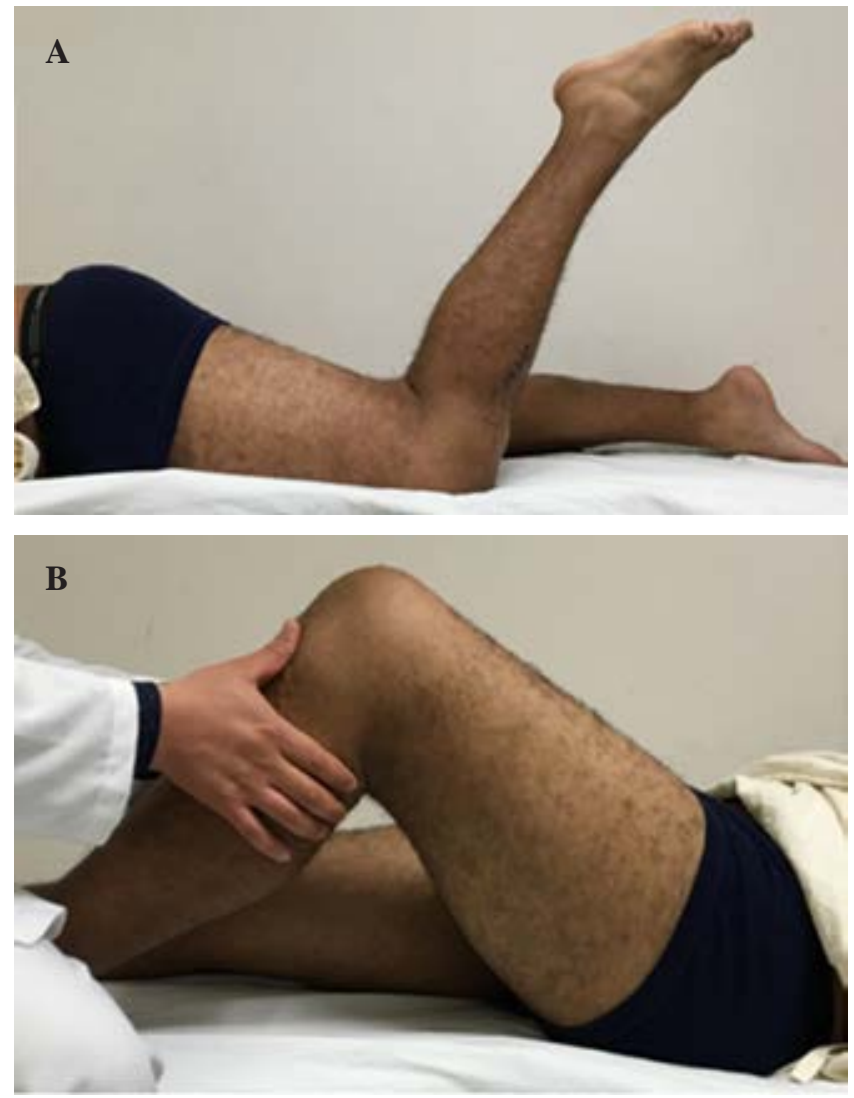

Figura 4: A) Se observa limitación a la flexión (75 grados). B) Test del cajón posterior negativo.

La reparación de tendón patelar fue término-terminal con sutura tipo Krackow modificado con poli (tereftalato de etileno) del número 5, se colocó para protección un tensor de Muller (Figura 2). Por último, se recolocó fijador externo para proteger la plastía ligamentaria durante dos semanas (Figura 2). Para iniciar la movilización del paciente se colocó rodillera mecánica a 0 grados de extensión, la cual se va flexionando paulatinamente a 15 grados el primer mes, a 30 grados a los dos meses, a 90 grados a los tres meses y se retiró de la misma al cuarto mes.

Para valorar la función de la articulación utilizamos la escala 2000 IKDC (International Knee Documentation Committee para la evaluación subjetiva de la rodilla) ${ }^{11}$ encontrando al paciente con un puntaje de 88.5 ocho meses después del tratamiento quirúrgico definitivo. ${ }^{11}$

\section{Discusión}

En este caso se observa lesión del sistema estabilizador de la rodilla, el cual se reporta en la literatura con una incidencia de $<10: 10,000 .{ }^{2}$ Constituye menos de $0.02 \%$ de todas la lesiones ortopédicas. ${ }^{3}$ Esta lesión se asocia además a la ruptura del tendón patelar, la cual es documentada con una prevalencia de $2.4 \% .{ }^{4}$ Se debe recalcar que la lesión estuvo asociada a luxación expuesta, la cual se encuentra con una frecuencia que varía de $9^{6}$ a 20 a $35 \% .^{7}$
El diagnóstico inicial se estableció a visión directa en el primer tiempo quirúrgico. Sin embargo, debemos tener en cuenta que en la bibliografía revisada se debe seguir un protocolo completo que consta de exploración física, radiografías, ecografía Doppler y resonancia magnética. El protocolo se completó para llegar al diagnóstico definitivo lesión multiligamentaria de rodilla estadio V de Schenck ${ }^{12,13}$ asociada a ruptura de tendón patelar. Se descartó lesión vascular y lesión nerviosa que se presentan con una frecuencia de $23-32$ y $14-40 \%$ respectivamente, ${ }^{5}$ lo cual influye de manera directa en la evolución y recuperación del paciente.

Para control de daños se hizo lavado mecánico, químico y desbridamiento así como estabilización de la extremidad con fijador externo. El tratamiento quirúrgico definitivo después de descartar infección mediante estudios de imagen y laboratorio se realiza en un solo tiempo quirúrgico después de cuatro semanas. Consideramos que esto corresponde a una reconstrucción en fase aguda, ya que algunos autores clasifican estas lesiones como reconstrucciones en fase aguda que se realizan dentro de las dos o tres semanas después de la lesión; ${ }^{10}$ otros autores citan como fase aguda a aquéllas que se realizan dentro de las seis semanas después de la lesión. ${ }^{14}$ Se prefiere efectuar la cirugía dentro de las seis semanas después de la lesión, ya que se disminuye el riesgo de inestabilidad residual, aunque se aumenta el riesgo de artrofibrosis. Moatshe informó sobre 303 pacientes con luxaciones de rodilla asociadas a lesión multiligamentarias no asociadas a ruptura de tendón rotuliano, donde el porcentaje de pacientes que desarrollaron artrofibrosis fue de $15.2 \%$ en los tratados en la fase aguda y de $3.8 \%$ en los tratados después de tres semanas. ${ }^{5}$ Geeslin notifica que los pacientes tratados en fase aguda (seis semanas después de la lesión) presentan una estabilidad articular significativamente mejor. ${ }^{14}$ Por lo tanto, Moatshe prefiere el tratamiento agudo de las estructuras lesionadas para facilitar la rehabilitación temprana, ya que es más fácil tratar la rigidez que la inestabilidad. ${ }^{5}$

Con el tratamiento quirúrgico buscamos restablecer el aparato extensor, flexor y lograr una rodilla funcional y estable. En nuestro paciente logramos a los ocho meses de realizar el evento quirúrgico definitivo un puntaje de 88.5 con el formulario IKDC $2000^{11}$ para la evaluación funcional subjetiva de la rodilla. Con esto se pudo integrar a las actividades de su vida diaria transcurrido este período (Figura 3). Sin embargo, observamos limitación a la flexión (Figura 4), lo cual concuerda con la literatura, ya que refiere que pacientes con lesión de ligamento cruzado anterior y posterior presentan tasas más altas de déficit de flexión, mayores índices de inestabilidad posterior y menores tasas de retorno a los niveles de actividad previos a la lesión. ${ }^{5}$ A pesar de lo mencionado, nuestro paciente no mostró inestabilidad posterior (Figura 4) y pudo retornar a los niveles de actividad previos.

\section{Conclusión}

La lesión multiligamentaria de rodilla asociada a ruptura de tendón patelar es poco frecuente. El tratamiento quirúr- 
gico se efectuó entre tres y seis semanas después de la lesión inicial. Se concluye que en etapa aguda se incrementa el riesgo de artrofibrosis; sin embargo, es preferible tratar la artrofibrosis que la inestabilidad.

Referencias

1. Mueller TB, O’Brien L. Multiligament knee injuries in athletes, is it possible to return to play? A rehabilitation perspective. Ann Joint. 2018; 3: 92. Available in: http://dx.doi.org/10.21037/aoj.2018.11.06.

2. Ali A, Abdelwahab M. Short-term outcome of multi-ligament knee injury among sudanese patients. Open Access Maced J Med Sci. 2019; 7(9): 1486-93.

3. Verma N, Singh H, Srivastav S. Multiligamentous knee injury e A Level III study to describe systematic approach, management and rehabilitation protocol with review of literature. Journal of Arthroscopy and Joint Surgery. 2018; 30: 1-7.

4. Malvasi S, Gloyeske B, Johnson M, Miller T. Multi-ligamentous knee injury in sports involving concomitant anterior cruciate ligament and patellar tendon disruption: a review of case reports. International Journal of Athletic Therapy \& Training. 2016; 2(3): 24-7.

5. Moatshe G, Chahla J, LaPrade R, Engebretsen L. Diagnosis and treatment of multiligament knee injury: state of the art. Journal of ISAKOS. 2017; 2: 152-61. Sitio web: \#BIBL http://jisakos.bmj.com/ content/early/2017/03/07/jisakos-2016-000072.
6. Lustig S, Leray E, Trojani C. Dislocation and bicruciate lesions of the knee: epidemiology and acute stage assessment in a prospective series. Orthop Traumatol Surg Res. 2009; 95(8): 614-20.

7. Gigante F, Lapera M, Zabala M. Conducta inicial en la luxación aguda de rodilla. Nuestra experiencia de 20 años. Artroscopia. 2016; 23: 61-9.

8. Aguirre RVH, Hernández TM, Valero GFS. Lesión multiligamentaria de rodilla. Acta Med. 2019; 17(2): 150-4.

9. Benazzo F, Zanon G, Perticarini L. Knee: multiligament injuries. Football Traumatology: New Trends. 2015; 19: 191-9.

10. Messer M, Southam B, Grawe B. Bilateral multiligamentous knee injuries: a case report and technique review. Case Rep Orthop. 2018; 2018: 3460153.

11. Arcuri F, Abalo E, Barclay F. Uso de escores para evaluación de resultados en cirugía del ligamento cruzado anterior. Artroscopia. 2010; 17(3): 241-7. Disponible en: https://www.revistaartroscopia. com/ediciones-anteriores/2010/volumen-17-numero-3/64-volumen05-numero-1/volumen-17-numero-3/606-uso-de-escores-paraevaluacion-de-resultados-en-cirugia-del-ligamento-cruzado-anterior.

12. Obremskey W, Molina C, Richards J. Early surgical treatment of highgrade multiligamentous knee injuries. Techniques in Orthopaedics. 2019; 34 (1): 58-68.

13. Buyukdogan K, Laidlaw M, Miller M. Surgical management of the multiple-ligament knee injury. Arthrosc Tech. 2018; 7(2): e147-64.

14. Geeslin A, LaPrade R. Outcomes of treatment of acute grade-III isolated and combined posterolateral knee injuries a prospective case series and surgical technique. J Bone Joint Surg Am. 2011; 93(18): 1672-83. 